\title{
Periodicities in rotation and DM of PSR B1557-50
}

\author{
A. E. Chukwude ${ }^{1,2}$, A. A. Ubachukwu ${ }^{2}$, and P. N. Okeke ${ }^{2}$ \\ ${ }^{1}$ Hartebeesthoek Radio Astronomy Observatory, PO Box 443, Krugersdorp 1740, South Africa \\ e-mail: austine@hartrao.ac.za; aus_chukwude@yahoo.com \\ 2 Department of Physics and Astronomy, University of Nigeria, Nsukka Enugu State, Nigeria \\ e-mail: EPSEELON@aol.com
}

Received 15 November 2001 / Accepted 11 November 2002

\begin{abstract}
We have analysed the pulse arrival time data of the pulsar B1557-50 collected over a period of nearly 13 years, from 1986 July to 1999 May, in order to investigate the long-term spin behaviour. The results reveal sustained cyclic variations in both the timing residuals and dispersion measure (DM) of this object. Both the timing and DM sequences are well described by two harmonically-related periodicities of $\sim 1600$ and 800 days. The variations in spin-down rate and DM are significantly anticorrelated in the sense that maximum $\dot{v}$ corresponds to minimum DM. Interpretation of the data in terms of free precession of an isolated radio pulsar suggests a wobble angle $\theta \approx 0.01^{\circ}$. The data are consistent with at least $6.5 \%$ of the stellar moment of inertia being coupled to the crust.
\end{abstract}

Key words. pulsars: general - pulsars: individual: B1557-50

\section{Introduction}

All isolated pulsars, except those residing in globular clusters, are expected to spin-down steadily with time owing to the drain on their rotational kinetic energy via magnetic dipole radiation and particle acceleration. In the context of the standard model (Manchester \& Taylor 1977), pulsar spin-down should be well described by a simple power-law relation of the form

$\dot{v}=-C v^{n}$,

where $v$ and $\dot{v}$ are the pulsar spin frequency and frequency derivative respectively, $n$ is the braking index and $C$ is a positive constant which depends on the magnetic moment and moment of inertia of the neutron star. Deviations from the simple spin-down law have been observed in the form of glitches - sudden and irregular jumps in pulsar spin frequency and its derivative (Wang et al. 2000, and references therein) and timing noise characterized by continuous, random fluctuations in pulsar spin rates (D'Alessandro et al. 1995, and references therein). Glitches and timing noise are both irregular events and appear to be more pronounced in young and middle-age pulsars (Cordes \& Downs 1985; Urama \& Okeke 1999).

However Stairs et al. (2000) and Shabanova et al. (2001) have recently presented strong evidence for the existence of sustained systematic form of deviations from the pulsar smooth spin-down law, resulting in a periodic or quasi-periodic pattern in the timing residuals of few pulsars. The variations have been linked to dynamical changes intrinsic to the pulsar, the leading candidate being the precession of isolated neutron stars

Send offprint requests to: A. E. Chukwude, e-mail: aus_chukwude@yahoo.com
(Link \& Epstein 2001). To date, only the observed cyclic variations in the timing residuals of two pulsars - PSR B1828-11 (Stairs et al. 2000) and PSR B1642-03 (Cordes 1993; Shabanova et al. 2001) - have been firmly linked to modulation associated with a precessing neutron star. Free precession interpretation of the data on these two pulsars was made unambiguous by the detection of correlated periodic variations in the observed pulse profile morphology, probably as the line-ofsight to the earth cuts through different sections of the pulsar's radiation beam.

It is most unlikely that the phenomenon of free precession is limited to these two pulsars, hence the need to extend the search for evidence of precession to more radio pulsars. Further observations of more precessing pulsars are required in order to shed more light on the poorly understood physics of free precession, especially on how different components of a neutron star respond to precession torque and on the role of superfluid pinning in precession.

Here we report the observation of a sustained cyclic behaviour in both the timing residuals and dispersion measure of the pulsar B1557-50, possibly suggesting the discovery of another precessing pulsar. The pulsar was discovered in the first Parkes pulsar survey (Komesaroff et al. 1973). It is relatively young, with a characteristic age of $0.60 \mathrm{Myr}$, and has a surface magnetic field of $1.0 \times 10^{12} \mathrm{G}$.

\section{Observations}

PSR B1557-50 is one of the 28 pulsars that have been routinely monitored at the Hartebeesthoek Radio Astronomy Observatory (HartRAO) since 1984 January. However, observations of PSR B1557-50 only commenced in July 1986 
and was continuous until May 1999. Observations were interupted between June 1999 and December 2000, after which continuous observing recommenced. Measurements of pulse time-of-arrival were made fortnightly with the 26 - $\mathrm{m}$ radio telescope at either 1668 or $2272 \mathrm{MHz}$. The observing bandwidth was fixed at $10 \mathrm{MHz}$ at both frequencies, but no pre-integration dedispersion hardware was available. Dispersive pulse broadening amounted to 1.8 and $4.6 \mathrm{~ms}$ for observations at 2272 and $1668 \mathrm{MHz}$ respectively. These values were assumed constant, since no major changes had been made to the observing hardware, and were subtracted in subsequent data processing.

The pulses were smoothed with a $0.3 \mathrm{~ms}$ time-constant filter, sampled at $\sim 0.113 \mathrm{~ms}$ interval and integrated over 4670 pulsar periods. An observation consisted of three such on-line integrations. Integration was initiated on a particular second by synchronization to the station clock, which was derived from a hydrogen maser and was referenced to UTC via GPS. A topocentric arrival time was obtained from each observation by least-squares fitting an analytical pulse model to the data. The model was a Gaussian whose parameters were derived from the pulse profile template, which is an accumulation of a large number of high signal-to-noise ratio profiles. Typical TOA uncertainties and signal-to-noise ratio are $0.2 \mathrm{~ms}$ and 5, respectively. Details of data acquisition and reduction at HartRAO have been described elsewhere by Flanagan (1993).

\section{Timing analysis and results}

Topocentric arrival times obtained at HartRAO were transformed to arrival times at infinite observing frequency at the solar system barycentre using the Jet Propulsion Laboratory DE200 ephemeris of the solar system and TEMPO software package (http://pulsar.princeton.edu/tempo). Subsequent model fitting analysis was implemented with the Observatory's software which is based on standard pulsar timing technique (Manchester \& Taylor 1977) and is well described by Flanagan (1995).

The time residuals, defined in the sense of model-predicted minus observed arrival times, were obtained by fitting the barycentric arrival times for the pulsar with a second-order polynomial to account for the secular pulsar spin-down. The residuals were used to obtain improved estimates of both rotational and astrometric parameters. The timing residuals of the pulsar over the $\sim 13$-year period are shown in Fig. 1 while the resulting timing and astrometric parameters are summarized in Table 1.

The presence of a cyclic pattern in the pulsar rotation is evident in Fig. 1. No fewer than three cycles, whose amplitudes vary between 4 and $8 \mathrm{~ms}$, with peak-to-peak separation of about 1600 days, are evident in the data. It is most unlikely that the observed modulation of the pulsar period is an artefact of our data reduction method. The same method was used to analyse the timing data on a sample of 28 HartRAO pulsars but only three objects were found to exhibit a credible cyclic pattern in their timing residuals over the entire data span.
Table 1. Measured parameters of PSR B1557-50.

\begin{tabular}{ll}
\hline \hline Parameter & Value $^{\mathrm{a}}$ \\
\hline Period, $P(\mathrm{~s})$ & $0.1926012327811(7)$ \\
Period derivative, $\dot{P}\left(10^{-15} \mathrm{ss}^{-1}\right)$ & $5.062326(8)$ \\
Epoch of period (MJD) & 49215.5 \\
Right ascension, $\alpha(J 2000.0)$ & $16^{\mathrm{h}} 00^{\mathrm{m}} 53^{\mathrm{s}} .031(3)$ \\
Declination, $\delta(J 2000.0)$ & $-50^{\circ} 44^{\prime} 20^{\prime} \cdot 96(6)$ \\
$D M\left(\mathrm{~cm}^{-3} \mathrm{pc}\right)$ & $260.56(9)$ \\
\hline
\end{tabular}

${ }^{\text {a }}$ Errors refer to the least significant digit and are $2 \sigma$ formal standard errors.

\subsection{Analysis of the spin-down and DM parameters of PSR B1557-50}

For a more detailed investigation of the observed cyclic variation in the rotation of the pulsar B1557-50, we examine the time evolution of the spin-down parameters: the rotation frequency $(v)$, frequency derivative $(\dot{v})$ and the dispersion measure $(D M)$. Series of $v(t), \dot{v}(t)$ and $D M(t)$ were calculated from local fits of second-order polynomial model plus a $D M$ term to short segments of data, whose lengths vary between 170 and 240 days. The choice of segment lengths was guided by the desire to keep the uncertainties in $\dot{v}(t)$ at reasonably the same level and to restrict the number of data points at 13 and $18 \mathrm{~cm}$ to $\geq 9$ at each wavelength per segment.

The residuals of the frequency $\Delta v(t)$ and frequency derivative $\Delta \dot{v}(t)$ and dispersion measure $\Delta D M(t)$ are shown in Fig. 1 . The timing residuals show some evidence of timing noise activity, in the form of microglitches. The sudden slope change in time residuals at about MJD 47945 suggests, on further analysis, a small glitch in $v$ and $\dot{v}$ whose amplitude is $\Delta v / v=$ $-9 \pm 2 \times 10^{-10}$ and $\Delta \dot{v} / \dot{v}=1.5 \pm 0.2 \times 10^{-4}$. The size and signature of this event are typical of timing noise behaviour (D'Alessandro et al. 1995 and references therein). In addition, a number of other significant scatters in $\Delta v(t)$ and $\Delta \dot{v}(t)$ could also be attributed to this microglitch activity.

However, the dominant feature, by far, in the time sequences $-\Delta t, \Delta v(t), \Delta \dot{v}(t)$ and $\Delta D M(t)-$ is sustained cyclic variations. The variations are reasonably periodic and are characterized by peak-to-peak spacing of about 1600 days, with the observed changes in $\dot{v}$ amounting to $\sim 0.08 \%$ of its mean value of $1.364 \times 10^{-13} \mathrm{~Hz} / \mathrm{s}$. A combination of two sinusoids, whose frequencies are in 1:2 ratio provides a very good fit to the current data. There exists a rather weak trend in Fig. 1d which suggests an overall linear increase in $D M$ with time. This change could be attributed to propagation effects of interstellar medium. Nonetheless the most dominant feature, by far, in the $D M$ time series is the cyclic variations, which are reasonably related to the changes in $\dot{v}(t)$ in the sense that maximum $\dot{v}$ corresponds to minimum $D M$.

For the purposes of finding the periodicities contained in both the timing and DM sequences of PSR B1557-50, spectral analysis of the data was performed using the Fourier transform technique (Scargle 1982). In view of the overall linear trend in the $\triangle D M$ data, it was necessary to first detrend the data, by subtracting a linear slope from the $D M$ times series, before taking the periodogram. The result of the analysis, summarized 


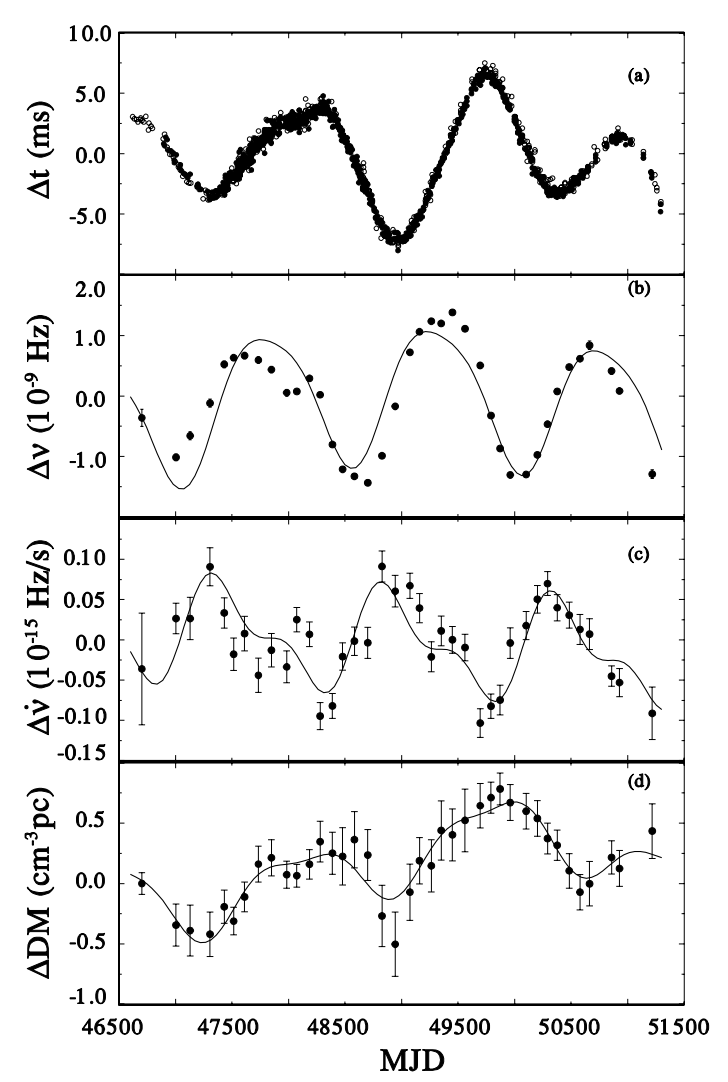

Fig. 1. Variations in the spin parameters and $D M$ of PSR 1557-50 over a 13 -year period. a) The timing residuals $\Delta t$ over the interval from 1986 to 1999 after accounting for the spin-down models listed in Table 1. b), c) and d) The time, frequency, frequency derivative and $D M$ residuals $(\Delta v, \Delta \dot{v}$ and $\Delta D M)$ after subtracting a model $[v, \dot{v}]$ plus $D M$ of length $4670 \mathrm{~d}$ from the $v(t), \dot{v}(t)$ and $D M(\mathrm{t})$ data obtained as described in the text. The solid curves are the predictions of two sinusoids with periodicities of 1600 and 800 days fitted to $\Delta \dot{v}$. The amplitudes of the $D M$ curves were obtained from a direct fit of 2 sinusoids with periods of 1600 and 800 days to the $D M$ data. Error bars are $2 \sigma$ formal standard errors.

in Fig. 2, reveals the presence, in all the time sequences, of a strong Fourier power at a frequency of $\sim 0.00063 \mathrm{~d}^{-1}$ with a possibility for a second, but much weaker, Fourier power at a frequency of about $0.0013 \mathrm{~d}^{-1}$. The periodicities corresponding to these frequencies are $\sim 1600$ and $800 \mathrm{~d}$ for 0.00063 and $0.0013 \mathrm{~d}^{-1}$ respectively.

\subsection{Analysis of the dual-frequency observations of PSR B1557-50}

Another unique feature of the current HartRAO timing data is that observations were regularly alternated between the two frequencies of 1.668 and $2.272 \mathrm{GHz}$. The timing residuals of these dual frequency observations for PSR B1557-50, as shown in Fig. 3a, reveal no apparent difference in the general form of the high- and low-frequency residual curves. To determine whether there exists an appreciable time offset between the two residual curves at some phases, we employed the method introduced by Shabanova et al. (2001) in a similar analysis. The time offsets were calculated by subtracting the low-frequency timing

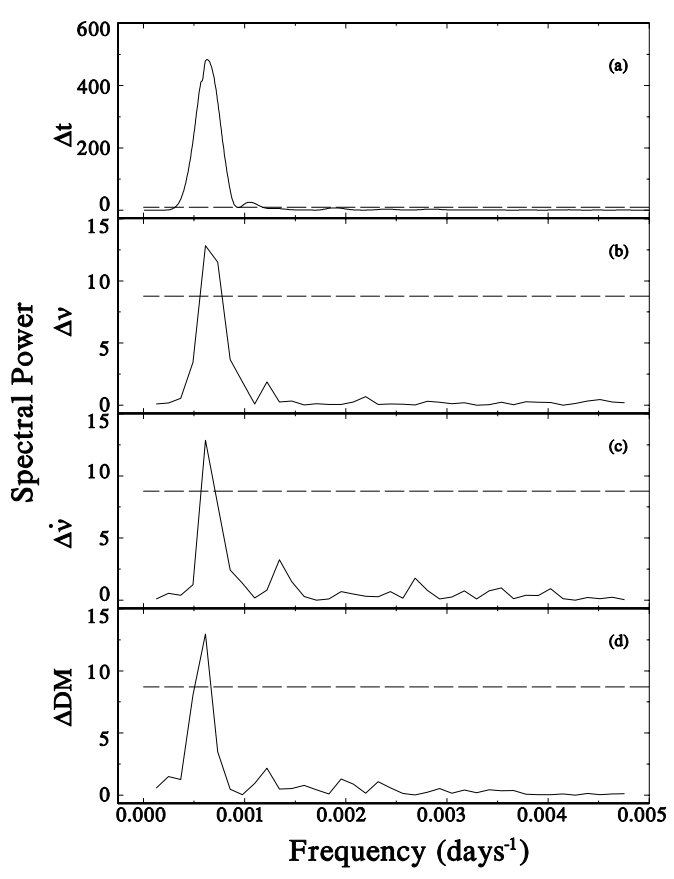

Fig. 2. Power spectrum of a) the time residuals $\Delta t$, b) the frequency residuals $\Delta v, \mathbf{c})$ the frequency derivative residuals $\Delta \dot{v}$ and $\mathbf{d}$ ) the DM residuals $\triangle D M$ obtained using the Fourier transform technique. The dashed horizontal lines represent the 3- $\sigma$ detection threshold (Scargle 1982: Eq. (18)).

residuals from the high-frequency timing residuals, averaged over independent bins of length $\sim 30$ days. The resulting time offsets, calculated from only bins containing observations at both 1.668 and $2.272 \mathrm{GHz}$, are shown in Fig. $3 \mathrm{~b}$ while the dual frequency timing residuals from which the time offsets were calculated are shown in Fig. 3a. Superimposed on the large scatter in the data are weak quasi-sinusoidal variations with maximum amplitude of $\sim 0.5 \mathrm{~ms}$. This quasi sine wave is almost out of phase with the time residual curves: in the sense that at minimum of the time residuals, the $1.668 \mathrm{GHz}$ pulses arrive $\sim 0.5 \mathrm{~ms}$ later than the pulses at $2.272 \mathrm{GHz}$. A similar observation has been recently reported by Shabanova et al. (2001) for the, presumably, precessing pulsar B1642-03, and the explanation most probably lies in the pulse shape variations at the two frequencies owing to changes in the time alignment of the low- and high-frequency pulse profiles during each precession cycle.

\section{Discussion}

A possible interpretation of the current observations on PSR B1557-50 is in terms of the presence of planetary system in orbit with the pulsar. The reflex motion of the pulsar about the common center of mass of the pulsar planet system would naturally introduce a cyclic modulation of the timing residuals, as has been observed for the pulsar B1257-12 by Wolscszan \& Frail (1992). The current data is not totally inconsistent with there being a $\sim 1.5$ Earth-mass planet in a nearly circular Keplerian orbit of radius $3 \mathrm{AU}$ with an orbital period of about 1600 days. However, we believe the observations severely constrain the planetary interpretation in 


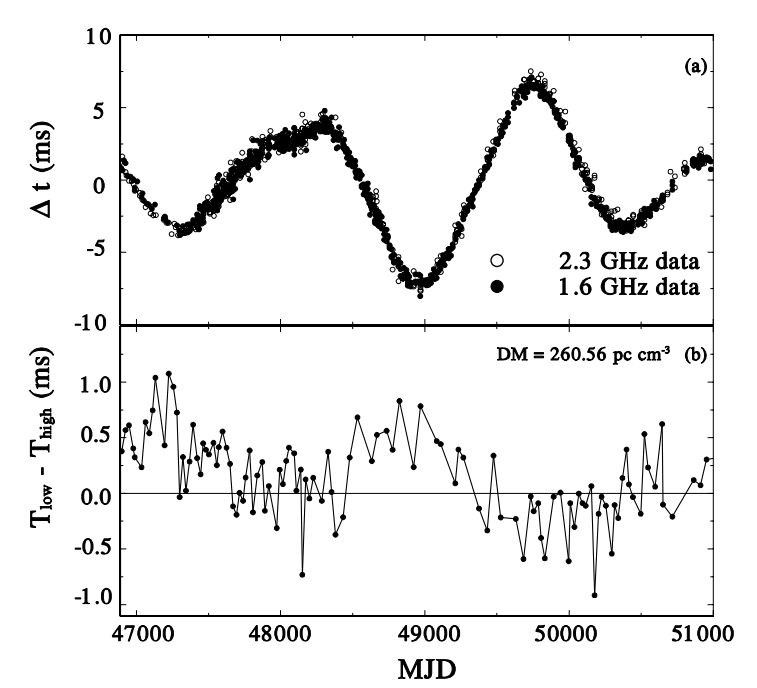

Fig. 3. a) Dual-frequency timing residuals of PSR B1557-50 between MJD 46890 and 51000. b) Time averaged differences in the timing residuals obtained at low frequency of $1.6 \mathrm{GHz}$ and at high frequency of $2.3 \mathrm{GHz}$.

at least three ways. Firstly a direct fit of a hypothetical planet to the timing data could not describe the data particularly well, improving the reduced chi-square by less than a factor of 1 . No significantly better fit to the data was possible by including two or more such planets. Moreover, it has remained an outstanding problem to find a physically plausible scenario that would explain the formation of planets around relatively young pulsars. Most importantly, the observed quasi-sinusoidal variations in both the $D M$ and the time offset between the low- and high-frequency timing data are difficult to explain in the context of the planetary scenario. Alternative explanation of the present observation in terms of timing noise - either in the form of random walks (Boynton et al. 1972) or some large-scale internal oscillations of neutron star (Ruderman 1970) - has difficulties in accounting for both the sustained regular cyclic pattern and the observed periodicities in the data. Furthermore, it is highly improbable that timing noise of any sort could plausibly explain the observed variations in both the $D M$ and the dual-frequency residual offsets.

The most physically plausible explanation of the current data lies in the precession of an isolated neutron star (Shaham 1977; Cordes 1993; Jones \& Andersson 2001). An isolated pulsar could precess if the underlying neutron star has a nonspherical shape and there is a misalignment of its spin and symmetry axes. Precession will introduce a cyclic variation in the pulsar spin-down torque as $\alpha$, the angle between its spin and magnetic axes, changes over the precession cycle. Such motion would certainly produce observable periodic or, atleast, quasi-periodic modulation in the amplitude of the timing residuals at the precession frequency (Jones \& Andersson 2001) and, possibly, in the pulse profile (Nelson et al. 1990). Theoretically (Cordes 1993; Jones \& Andersson 2001), an important parameter of free precession is the wobble angle $(\theta)$, an offset angle between the neutron star spin and symmetry axes, on which the amplitudes of both the timing and pulse profile modulation depend. Recently, Jones \& Andersson (2001) and
Link \& Epstein (2001) have undertaken extensive modelling of free precession phenomenon in isolated neutron stars. One of the conclusions reached, independently, by Link \& Epstein; Jones \& Andersson is that the phase modulation in a precessing pulsar will be characterized by two frequencies in 1:2 ratio if the dipole moment axis of the star is nearly orthogonal to its symmetry axis (i.e. $\chi \approx \pi / 2$ ). The spectral analysis of both the timing and DM residuals for PSR B1557-50 reveal excess power at the frequencies of 0.00063 and $0.0013 \mathrm{~d}^{-1}$, which are in 1:2 ratio, corresponding to periodicities of 1600 and $800 \mathrm{~d}$ respectively. However, although the second frequency at $\sim 0.0013 \mathrm{~d}^{-1}$ is very suggestive, we remark that it is rather highly speculative for some reasons: the peak fails even the $1-\sigma$ level detection probability and Scargle periodograms are known to show artifacts at this level. However, using the dominant periodicity of $1600 \mathrm{~d}$, the observed $\sim 6 \mathrm{~ms}$ amplitude modulation in the phase residuals of PSR B1557-50 over the 13 -year period suggests a wobble angle $\theta \approx 1.4 \times 10^{-4} \tan \chi \mathrm{rad}$, Eq. (65) of Jones \& Andersson (2001). In general, $0 \leq \chi \leq$ $90^{\circ}$, though the exact value is poorly known for most pulsars. However, adopting a value of $49^{\circ}$ published for this object by Kuz'min et al. (1984), we obtain $\theta \approx 0.01^{\circ}$.

Precession would naturally produce variations in the observed pulse profile, as the observer views the pulsar beam from different angles over the precession cycle. Such variations in the pulse profile have been investigated in the past using the pulse shape parameters (e.g. Cordes 1993; D'Alessandro \& McCulloch 1997; Stairs et al. 2000) and are expected to correlate with the variations in the pulsar rotation. However, it is most likely that the observed cyclic variations in the $D M$ of PSR B1557-50, which are significantly $(\sim 70 \%)$ anti-correlated with changes in pulsar spin-down rate are directly linked to variations in the pulsar spin-down torque owing to precession. Furthermore, a combination of two sinusoids, with periodicities in 2:1 ratio, obtained from a fit to $\Delta \dot{v}(t)$ data offers a particularly good description of the $D M$ data. A plausible interpretation of this long-term $D M$ variations is in terms of the changes in observed pulse profile shape. A possible scenario is that a change in the line-of-sight cut across the emission region results in a more significant pulse shape change at one frequency than the other. Consequently, there would be changes in the effective time alignment of the two pulse profiles which would affect the arrival times of the pulses at 2.272 and $1.668 \mathrm{GHz}$ appreciably differently. The observed $\sim 0.5 \mathrm{~ms}$ weak quasi-sinusoidal modulation of the time offset between the low- and high-frequency timing data further supports the current interpretation of the long-term $D M$ variations. Similar quasi-sinusoidal modulation of the time offset between lowand high-frequencies arrival times was recently reported for the precessing pulsar B1642-03 by Shabanova et al. (2001), who explained it in terms of differences in the observed pulse profiles at the two frequencies or small amplitude change in the pulsar dispersion measure. In the case of PSR B1557-50, the $\sim 0.5 \mathrm{~ms}$ delay in the arrival times at 1.668 and $2.272 \mathrm{GHz}$ suggests a change in DM of $\sim 0.35 \mathrm{pc} \mathrm{cm}^{-3}$, which is not at serious variance with the observed $\sim 0.5 \mathrm{pc} \mathrm{cm}^{-3}$ amplitude modulation in the DM variations (Fig. 1d). In the case of the pulsar B1557-50, the data do not reveal any measureable long-term 
pulse shape variations at either of the two frequencies, probably owing to the expected low amplitude wobble of the beams and the low signal-to-noise ratio of the current data.

The response of the different components of a neutron star to the precession torque is still poorly understood. Glitch observations (Flanagan 1990; McCulloch et al. 1990) suggest the pinning of a few percent of the stellar moment of inertia to the inner crust via superfluid vortices, a situation which would limit the precession period to $\sim 100 P$, where $P$ is the rotation period of the pulsar, (Shaham 1977; Sedrakian et al. 1999). However acceptance of the long periodicities observed in some pulsars as evidence for free precession places a severe limit on the fraction of superfluid vortices that are actually pinned to the inner crust. Jones and Andersson (2001) have shown that the data for the two known precessing pulsars, B1828-11 and B1642-03, are consistent with zero pinning of superfluid vortices, with an upper limit on ratio of the moment of inertia of pinned superfluid to the total stellar moment of inertia of $10^{-10}$. Assuming zero superfluid pinning and that only the crust participates in the precession, the 1600-day modulation in PSR B1557-50 yields a reference oblateness, $\epsilon_{0}=1.4 \times 10^{-6}$. This is smaller than the actual oblateness due to rotational deformation, $\epsilon_{\text {fluid }}=5.7 \times 10^{-6}$. Thus Eq. (39) of Jones \& Andersson (2001) is violated, implying that more than just the crust participates in the precession. To fit the present data on PSR B1557-50 would require $I_{0}=0.065 I_{\text {tot }}$, where $I_{0}$ is the moment of inertia of the crust and all components coupled to it and $I_{\text {tot }}$ is the moment of inertia of the entire neutron star. This suggests that, at least, $6.5 \%$ of the total stellar moment of inertia need to participate in the free precession in order to increase the $\epsilon_{0}$ to the minimum acceptable value of $\epsilon_{\text {fluid }}$ required to satisfy the inequality (39) of Jones \& Andersson (2001). However, even for the extreme case of strong coupling, in which all the neutron star components are responding to the precession torque, we still find that the pinned superfluid component cannot constitute more than $1.4 \times 10^{-9}$ of the stellar moment of inertia, several orders of magnitude less than 0.01 obtained from glitch observations (e.g. Flanagan 1990), and is consistent with $\sim 10^{-10}$ found for the pulsars B1828-11 and B1642-11 (Jones \& Andersson 2001).

\section{Conclusion}

We have investigated the spin behaviour of the pulsar B1557-50 over a period of 13 years. The result shows sustained and very nearly periodic variations in both rotational and DM parameters of this object. A particularly good description of the data is given by a combination of two sinusoids with two harmonically-related frequencies of 0.00063 and $0.0013 \mathrm{~d}^{-1}$.
The observations presented here are most plausibly explained in terms of free precession of the pulsar B1557-50, requiring a wobble angle $\theta \sim 0.01^{\circ}$ and about $6.5 \%$ of the total stellar moment of inertia to be strongly coupled to the crust, and hence participates in the precession.

Acknowledgements. We thank Claire Flanagan and Johnson Urama for their helpful comments and discussions, and Mike Gaylard for critically reading through the manuscript. AEC acknowledges the support and hospitality of HartRAO Director, G.D. Nicolson. He is grateful to IAU Commission 38 for supporting his visit to HartRAO with a travel grant and to an anonymous referee for his useful comments which helped to improve this paper.

\section{References}

Boynton, P. E., Groth, E. J., Hutchinson, D. P., et al. 1972, ApJ, 175, 217

Cordes, J. M. 1993, in Planets around Pulsars, ed. J. A. Phillips, S. E. Thorsett, \& S. R. Kulkarni (San Francisco: ASP), ASP Conf. Ser., 36,61

Cordes, J. M., \& Downs, G. S. 1985, ApJS, 59, 343

D'Alessandro, F. D., \& McCulloch, P. M. 1997, MNRAS, 292, 879

D’Alessandro, F. D., McCulloch, P. M., Hamilton, P. A., et al. 1995, MNRAS, 277, 1033

Flanagan, C. S. 1990, Nature, 345, 416

Flanagan, C. S. 1993, MNRAS, 260, 643

Flanagan, C. S. 1995, Ph.D. Thesis, Rhodes University, Grahamstown, South Africa

Jones, D. I., \& Andersson, N. 2001, MNRAS, 324, 811

Komesaroff, M. M., Abels, J. G., Cooke, D. J., et al. 1973, ApJ, 15, L169

Kuz'min, A. D., Dagkesamanskaya, I. M., \& Pugachëv, V. D. 1984, Sov. Astron. Lett., 10, 6

Link, B., \& Epstein, R. I. 2001, ApJ, 556, 392

Manchester, R. N., \& Taylor, J. H. 1977, Pulsars (San Francisco: Freeman)

McCulloch, P. M., Hamilton, P. A., McConnell, D., et al. 1990, Nature, 346,822

Nelson, R. W., Finn, L. S., \& Wasserman, I. 1990, ApJ, 348, 226; Wasserman, I. 1990, ApJ, 348, 226

Ruderman, M. 1970, Nature, 225, 838

Scargle, J. D. 1982, ApJ, 263, 835

Sedrakian, A., Wasserman, I., \& Cordes, J. M. 1999, ApJ, 524, 360

Shabanova, T. V., Lyne, A. G., \& Urama, J. O. 2001, ApJ, 552, 321

Shaham, J. 1977, ApJ, 214, 251

Stairs, I. H., Lyne, A. G., \& Shemar, S. L. 2000, Nature, 406, 484

Urama, J. O., \& Okeke, P. N. 1999, MNRAS, 310, 313

Wang, N., Manchester, R. N., Pace, R. T., et al. 2000, MNRAS, 317, 843

Wolszczan, A., \& Frail, D. A. 1992, Nature, 355, 145 